\title{
Geometry and rank of fibered hyperbolic 3-manifolds
}

\author{
IAN BIRINGER
}

Recall that the rank of a finitely generated group is the minimal number of elements needed to generate it. In [22], M White proved that the injectivity radius of a closed hyperbolic 3-manifold $M$ is bounded above by some function of $\operatorname{rank}\left(\pi_{1}(M)\right)$. Building on a technique that he introduced, we determine the ranks of the fundamental groups of a large class of hyperbolic 3-manifolds fibering over the circle.

$57 \mathrm{M} 50$

\section{Introduction}

Let $\Sigma_{g}$ be the closed orientable surface of genus $g$ and $\phi: \Sigma_{g} \rightarrow \Sigma_{g}$ a homeomorphism. We can construct a 3-manifold $M_{\phi}$, the mapping torus of $\phi$, as the quotient space

$$
M_{\phi}=\Sigma_{g} \times[0,1] / \sim, \quad(x, 0) \sim(\phi(x), 1) .
$$

Thurston [19] has proven that if the map $\phi: \Sigma_{g} \rightarrow \Sigma_{g}$ is pseudo-Anosov then $M_{\phi}$ can be given a hyperbolic metric.

The fundamental group of $M_{\phi}$ is given by an $\mathrm{HNN}$-extension

$$
1 \rightarrow \pi_{1}\left(\Sigma_{g}\right) \rightarrow \pi_{1}\left(M_{\phi}\right) \rightarrow \mathbb{Z} \rightarrow 1 .
$$

Since $\operatorname{rank}\left(\pi_{1}\left(\Sigma_{g}\right)\right)=2 g$ it follows that $\operatorname{rank}\left(\pi_{1}\left(M_{\phi}\right)\right) \leq 2 g+1$. It is not hard to construct examples where this inequality is strict, but it seems likely that if the gluing map is complicated enough then equality should hold. As an illustration of this, J Souto proved in [18] that given a pseudo-Anosov map $\phi: \Sigma_{g} \rightarrow \Sigma_{g}$, we have for sufficiently large powers $\phi^{n}$ of $\phi$ that $\operatorname{rank}\left(\pi_{1}\left(M_{\phi^{n}}\right)\right)=2 g+1$. Our main result is the following extension of Souto's theorem.

Theorem 1.1 Given $\epsilon>0$ and a closed orientable surface $\Sigma_{g}$, there are at most finitely many $\epsilon$-thick hyperbolic 3-manifolds $M$ fibering over $S^{1}$ with fiber $\Sigma_{g}$ for which $\operatorname{rank}\left(\pi_{1}(M)\right) \neq 2 g+1$. 
Recall that the injectivity radius of a hyperbolic manifold $M$, written $\operatorname{inj}(M)$, is defined to be half the length of a shortest homotopically essential loop in $M$, and $M$ is called $\epsilon$-thick if $\operatorname{inj}(M) \geq \epsilon$.

It is worth noting that if $\epsilon$ is small then there are many hyperbolic 3-manifolds fibering over the circle that are $\epsilon$-thick. In fact, if $\phi_{1}, \ldots, \phi_{n}$ are pseudo-Anosov homeomorphisms of $\Sigma_{g}$, then for large $m$ the maps $\phi_{1}^{m}, \ldots, \phi_{n}^{m}$ freely generate a subgroup of $\operatorname{Mod}\left(\Sigma_{g}\right)$ all of whose elements are monodromies of mapping tori with a common lower bound on injectivity radius. This follows from combining work of Farb-Mosher [10, Theorem 1.4], Kent-Leininger [12, Theorem 1.2] and Rafi [15, Theorem 1.6]. It also demonstrates that Theorem 1.1 is strictly stronger than Souto's earlier result.

Results similar to Theorem 1.1 concerning the Heegaard genus of $M$ are already known; the strongest is due to Bachman and Schleimer [3]. Recall that the Heegaard genus of a closed 3-manifold $M$ is the smallest $g=g(M)$ such that $M$ can be obtained by gluing two genus $g$ handlebodies along their boundaries. It is easy to see that when $M$ fibers over the circle with fiber $\Sigma_{g}$ then $g(M) \leq 2 g+1$, and Bachman and Schleimer show that $g(M)=2 g+1$ as long as the monodromy map of $M$ has translation distance at least $2 g+1$ in the curve complex of $\Sigma_{g}$. It is likely that the conclusion of Theorem 1.1 is true under similar assumptions, but it is not yet clear to us how to prove this.

Before beginning the bulk of this paper, let us sketch the idea behind the proof of Theorem 1.1. Let $M$ be a hyperbolic 3-manifold fibering over the circle with fiber $\Sigma_{g}$. Following a technique of White [22], we find a graph $X$ with $\operatorname{rank}\left(\pi_{1}(X)\right)=\operatorname{rank}\left(\pi_{1}(M)\right)$ and a $\pi_{1}$-surjective mapping $f: X \rightarrow M$ whose image has as small length as possible. We show that if $M$ has large diameter it is most efficient for $X$ to use small edges to fill out the fundamental group of the fiber and a long edge to circumnavigate $M$ in the horizontal direction. The subgraph of $X$ consisting of all small edges then has rank at least $2 g$, since it generates a subgroup of $\pi_{1}(M)$ isomorphic to $\pi_{1}\left(\Sigma_{g}\right)$. But $X$ must have even larger rank, so $\pi_{1}(M)=\pi_{1}(X) \geq 2 g+1$.

The paper is organized as follows. We begin in Section 2 by recalling some standard facts from the theory of Kleinian groups. In Section 3, we use a lemma of Souto and a compactness argument to control the geometry of certain covers of doubly degenerate hyperbolic manifolds homeomorphic to $\Sigma_{g} \times \mathbb{R}$. The minimal length graphs mentioned above are formally introduced in Section 4, and Section 5 contains a proof of Theorem 1.1. We finish with an Appendix that fleshes out a result due to Souto [17] that gives a convenient decomposition for minimal length $\pi_{1}$-surjective graphs in closed hyperbolic 3 -manifolds. 
Acknowledgements I thank Justin Malestein, Nathan Broaddus and Benson Farb for their helpful comments, Lisa Wang for her help typesetting and Juan Souto for many conversations, advice and insight. Thanks are also due to the referee, who has greatly contributed to the accuracy and readability of this paper.

\section{Preliminaries}

Throughout the following, let $M$ be a hyperbolic 3-manifold with finitely generated fundamental group and no cusps. The resolution of Marden's Conjecture by Agol [1] and Calegari-Gabai [7] implies that $M$ is homeomorphic to the interior of a compact 3manifold $\bar{M}$. Consequently, the topological ends of $M$ are in bijective correspondence with the components of $\partial \bar{M}$, and each end $E$ has a neighborhood homeomorphic to $\partial E \times(0, \infty)$, where $\partial E$ is the component of $\partial \bar{M}$ that $E$ faces.

Since $M$ has no cusps, its ends admit a simple geometric classification. Specifically, recall that the convex core $\mathrm{CC}(M)$ is the smallest convex submanifold of $M$ whose inclusion is a homotopy equivalence; an end of $M$ is called convex-cocompact if its intersection with the convex core of $M$ is compact, and degenerate otherwise. The interested reader can consult Matuzaki and Tanaguchi [13] or Thurston [20] for a deeper investigation of this classification-we will limit ourselves here to presenting the prototypical example of a degenerate end, which is also the example most prevalent in the work to come.

Example 2.1 Let $M_{\phi}$ be the mapping torus of a pseudo-Anosov map $\phi: \Sigma_{g} \rightarrow \Sigma_{g}$. As mentioned in the introduction, $\pi_{1}\left(M_{\phi}\right)$ decomposes as

$$
1 \rightarrow \pi_{1}\left(\Sigma_{g}\right) \rightarrow \pi_{1}\left(M_{\phi}\right) \rightarrow \mathbb{Z} \rightarrow 1 .
$$

Let $N$ be the cyclic cover of $M_{\phi}$ corresponding to the subgroup $\pi_{1}\left(\Sigma_{g}\right)$. Then $N$ is homeomorphic to $\Sigma_{g} \times \mathbb{R}$, and since it regularly covers a closed manifold we have $\mathrm{CC}(N)=N$, implying that both ends of $N$ are degenerate. Note that unwrapping a fiber bundle structure for $M_{\phi}$ gives a product structure $N \cong \Sigma_{g} \times \mathbb{R}$ with fibers of bounded diameter, contrasting with the exponential growth of level surfaces in a convex-cocompact end.

\subsection{Simplicial hyperbolic surfaces}

We record here some facts about negatively curved surfaces in hyperbolic 3-manifolds.

Definition 2.2 Let $M$ be a hyperbolic 3-manifold. A simplicial hyperbolic surface in $M$ is a map $f: S \rightarrow M$, where 
- $S$ is a closed surface equipped with a triangulation $T$

- $f$ maps each face of $T$ to a totally geodesic triangle in $M$

- for each vertex $v \in T$ the angles between the $f$-images of the edges adjacent to $v$ sum to at least $2 \pi$.

If $f: S \rightarrow M$ is a simplicial hyperbolic surface then we get a path-metric on $S$ by requiring that $f$ preserves path lengths. The metric is smooth and hyperbolic away from the vertices of $T$, at which there are possible excesses of angle. By the GaussBonnet Theorem, we have $\operatorname{vol}(S) \leq 2 \pi|\chi(S)|$. Bounding the diameter of $S$ by its volume and injectivity radius, we obtain:

Bounded Diameter Lemma (Thurston) Assume $f: S \rightarrow M$ is an $\epsilon$-thick simplicial hyperbolic surface of genus $g$. Then $\operatorname{diam}(S) \leq \frac{4}{\epsilon}(2 g-2)$.

Mahler's Compactness Theorem [4, E.1] states that the moduli space of $\epsilon$-thick (smooth) hyperbolic surfaces of fixed genus is compact. Together with the following Proposition, this provides a number of upper bounds on the geometry of $\epsilon$-thick simplicial hyperbolic surfaces, albeit without explicit constants.

Proposition 2.1 (Smooth dominates simplicial) Let $S$ be a closed surface and $d$ a metric on $S$ that is the pullback metric for some simplicial hyperbolic surface. Then there exists a smooth hyperbolic metric $d_{\text {hyp }}$ on $S$ such that for all $x, y \in S$

$$
\frac{1}{C} d(x, y) \leq d_{\text {hyp }}(x, y),
$$

where $C>0$ depends only on the topological type of $S$. Note that if $d$ is $\epsilon$-thick then $d_{\text {hyp }}$ is $(\epsilon / C)$-thick.

Proof of Proposition 2.1 Working in polar coordinates in small neighborhoods around the singular points of $d$, we can explicitly deform $d$ to obtain a smooth metric $d^{\prime}$ with Gaussian curvature $K \leq-1$ that is bilipschitz to $d$ with bilipschitz constant depending only on the angles $d$ has around the points in its singular locus. The argument is very similar to the proof of the $2 \pi$-Theorem of Gromov and Thurston [5], so we will omit it here. Since the Gauss-Bonnet Theorem gives an upper bound for the sum of these singular angles, $d$ and $d^{\prime}$ are in fact $C$-bilipschitz for some $C$ depending only on the topological type of $S$. Define $d_{\text {hyp }}$ to be the hyperbolic metric in the conformal class of $d^{\prime}$. The Ahlfors-Schwartz Lemma [2] states that distances measured in $d^{\prime}$ are less than or equal to distances in $d_{\text {hyp }}$; this proves the desired inequality. 
As an application, we can use Proposition 2.1 and a based version of Mahler's Compactness Theorem to show:

Corollary 2.2 (Short markings) Set $\Gamma=\pi_{1}\left(\Sigma_{g}\right)$ and fix a generating set $X \subset \Gamma$. Then given $\epsilon, g>0$ there is a constant $L$ such that whenever $f: S \rightarrow M$ is an $\epsilon-$ thick simplicial hyperbolic surface of genus $g$ and $p \in S$, there is an isomorphism $\Phi: \Gamma \rightarrow \pi_{1}(S, p)$ such that the image of each element of $X$ can be represented by a loop based at $p$ of length less than $L$.

Observe that Corollary 2.2 is similar to Lemma 7.1 in [8], but it is slightly stronger and more easily applied in our work in Section 3.

\subsection{Algebraic and geometric convergence}

Let $\Gamma$ be a finitely generated group and consider a sequence of discrete and faithful representations $\rho_{i}: \Gamma \rightarrow \operatorname{PSL}(2, \mathbb{C})$. If $\left(\rho_{i}\right)$ converges pointwise to $\rho_{\infty}: \Gamma \rightarrow \operatorname{PSL}(2, \mathbb{C})$, we usually say that $\left(\rho_{i}\right)$ is algebraically convergent with $\rho_{\infty}$ as its algebraic limit. Alternatively, consider a sequence of subgroups $G_{i} \subset \operatorname{PSL}(2, \mathbb{C})$; if these converge to a subgroup $G \subset \operatorname{PSL}(2, \mathbb{C})$ in the Chabauty topology on closed subsets of $\operatorname{PSL}(2, \mathbb{C})$ then we say that $G_{i} \rightarrow G$ geometrically. The case where the two notions of convergence agree is useful enough to warrant additional terminology. Specifically, if $\rho_{i} \rightarrow \rho_{\infty}$ algebraically and $\rho_{i}(\Gamma) \rightarrow \rho_{\infty}(\Gamma)$ geometrically then one says that $\rho_{i} \rightarrow \rho_{\infty}$ strongly.

One can interpret the geometric convergence of a sequence of subgroups $G_{i} \rightarrow G_{\infty} \subset$ $\operatorname{PSL}(2, \mathbb{C})$ in terms of the quotient manifolds $M_{i}=\mathbb{H}^{3} / G_{i}$. If we fix a basepoint and baseframe $(p, f)$ for $\mathbb{H}^{3}$, for each $i$ we can take the projection $\left(p_{i}, f_{i}\right)$ as a basepoint and baseframe for $M_{i}$. Then $G_{i} \rightarrow G_{\infty}$ geometrically if there exist sequences of positive numbers $\epsilon_{i} \rightarrow 0$ and $R_{i} \rightarrow \infty$, and $\left(1+\epsilon_{i}\right)$-bilipschitz maps $\phi_{i}: \mathrm{B}\left(p_{i}, R_{i}\right) \rightarrow M_{\infty}$ sending $\left(p_{i}, f_{i}\right)$ to $\left(p_{\infty}, f_{\infty}\right)$. For future reference, we will call the maps $\phi_{i}$ a sequence of almost isometric maps coming from geometric convergence. Note that using this as our definition, we can speak about a geometrically convergent sequence of framed hyperbolic 3-manifolds, or even based hyperbolic 3-manifolds if we forget about the presence of a baseframe.

For a detailed study of algebraic and geometric convergence, see Matsuzaki and Tanaguchi [13] and Benedetti and Petronio [4].

\section{Short graphs in doubly degenerate $\Sigma_{g} \times \mathbb{R}$}

Assume that $M$ is a hyperbolic 3-manifold without cusps that is homeomorphic to $\Sigma_{g} \times \mathbb{R}$. Using Waldhausen's Cobordism Theorem [21] and Ahlfors' Finiteness 
Theorem [13], it is not hard to see that there is an explicit homeomorphism $M \cong \Sigma_{g} \times \mathbb{R}$ such that $\mathrm{CC}(M)$ sits inside $M$ as either

- $\quad \Sigma_{g} \times[0,1]$, in which case $M$ is convex cocompact

- $\Sigma_{g} \times[0, \infty)$, in which case $M$ is called singly degenerate

- $\Sigma_{g} \times \mathbb{R}$, and then $M$ is called doubly degenerate.

We mentioned in the introduction that Theorem 1.1 is an extension of an earlier theorem of Souto [18]. A key ingredient in Souto's proof was the following observation, which is a consequence of the Covering Theorem of Canary and Thurston [9]. It was originally proven by Scott-Swarup [16] in the case that $M$ is the cyclic cover of a hyperbolic 3 -manifold fibering over the circle.

Lemma 3.1 [18] Let $M$ be a doubly degenerate hyperbolic 3-manifold homeomorphic to $\Sigma_{g} \times \mathbb{R}$ and let $\Gamma \subset \pi_{1}(M)$ be a proper subgroup of rank at most $2 g$. Then $\Gamma$ is free, infinite index and convex-cocompact.

To prove Theorem 1.1, we need an improved version of Lemma 3.1 that gives a diameter bound for the convex core of $\mathbb{H}^{3} / \Gamma$ in terms of $\operatorname{inj}(M)$ and the length of a set of loops in $M$ generating $\Gamma$. Our proof will be a compactness argument: we define a topology on the set of wedges of $k$ bounded length loops in $\epsilon-$ thick doubly degenerate hyperbolic 3-manifolds homeomorphic to $\Sigma_{g} \times \mathbb{R}$, show that the resulting space is compact and then use continuity to show that there is an upper bound for the corresponding convex core diameters.

Definition 3.1 Define $\mathcal{G}=\mathcal{G}(\epsilon, L, k)$ to be the space of pairs $(M, f)$, where

(1) $M$ is a doubly degenerate $\epsilon$-thick hyperbolic 3-manifold homeomorphic to $\Sigma_{g} \times \mathbb{R}$

(2) $f: \bigwedge_{k} \mathbb{S}^{1} \rightarrow M$ is an $L$-Lipschitz map from the wedge of $k$ circles, endowed with some fixed metric.

We say that $\left(M_{i}, f_{i}\right) \rightarrow\left(M_{\infty}, f_{\infty}\right)$ if

(1) $\left(M_{i}, \star_{i}\right)$ converges strongly to $\left(M_{\infty}, \star_{\infty}\right)$, where $\star_{i}$ is the wedge point of $f_{i}\left(\bigwedge_{k} \mathbb{S}^{1}\right)$

(2) there is a sequence $\phi_{i}$ of almost isometric maps coming from the geometric convergence in (1) such that $\phi_{i} \circ f_{i}: \bigwedge_{k} \mathbb{S}^{1} \rightarrow M$ converges pointwise to $f_{\infty}: \bigwedge_{k} \mathbb{S}^{1} \rightarrow M_{\infty}$ 
Here, (1) means that there are faithful representations $\rho_{i}$ of $\pi_{1}\left(\Sigma_{g}\right)$ into $\operatorname{PSL}(2, \mathbb{C})$ which converge strongly to a representation $\rho_{\infty}$ such that the quotient manifolds $\mathbb{H}^{3} / \rho_{i}\left(\pi_{1}\left(\Sigma_{g}\right)\right)$ and $\mathbb{H}^{3} / \rho_{\infty}\left(\pi_{1}\left(\Sigma_{g}\right)\right)$ are isometric to $\left(M_{i}, \star_{i}\right)$ and $\left(M_{\infty}, \star_{\infty}\right)$ as based hyperbolic manifolds, where the projections of some fixed point in $\mathbb{H}^{3}$ are taken as basepoints.

\section{Proposition 3.2 $\mathcal{G}$ is compact.}

Proof Let $\left(M_{i}, f_{i}\right)$ be a sequence in $\mathcal{G}$ and assume that $\star_{i} \in M_{i}$ is the wedge point of $f_{i}\left(\bigwedge_{k} \mathbb{S}^{1}\right)$. For each $i$, Canary's Filling Theorem [9] gives a simplicial hyperbolic surface in $M_{i}$ with image passing through $\star_{i}$. Using the short markings of these surfaces provided by Corollary 2.2 we can construct representations $\rho_{i}: \pi_{1}\left(\Sigma_{g}\right) \rightarrow$ $\operatorname{PSL}(2, \mathbb{C})$ with $\mathbb{H}^{3} / \rho_{i}\left(\Sigma_{g}\right) \cong M_{i}$ so that a fixed base point $\star \in \mathbb{H}^{3}$ projects to each $\star_{i}$ and up to passing to a subsequence, $\rho_{i}$ converges algebraically to some $\rho_{\infty}: \pi_{1}\left(\Sigma_{g}\right) \rightarrow \operatorname{PSL}(2, \mathbb{C})$. Since our lower bound on injectivity radius persists through algebraic limits, $\rho_{\infty}\left(\pi_{1}\left(\Sigma_{g}\right)\right)$ contains no parabolics. Work of Thurston and Bonahon then implies that $\rho_{i} \rightarrow \rho_{\infty}$ strongly. Specifically, one must trace through Thurston's proof of $[20,9.2]$ with the hindsight provided by Bonahon's Tameness Theorem [6]. A statement of the resulting theorem is given by Canary in $[9,9.1]$ as a prelude to a series of more general convergence theorems.

Set $M_{\infty}=\mathbb{H}^{3} / \rho_{\infty}\left(\Sigma_{g}\right)$ and let $\star_{\infty} \in M_{\infty}$ be the projection of $\star$. Then $\left(M_{i}, \star_{i}\right)$ converges geometrically to $\left(M_{\infty}, \star_{\infty}\right)$. The fundamental group of $M_{\infty}$ is isomorphic to $\pi_{1}\left(\Sigma_{g}\right)$, so Bonahon's Tameness Theorem [6] implies that $M_{\infty} \cong \Sigma_{g} \times \mathbb{R}$. Moreover, it follows from strong convergence and [14, Theorem 1.1] that the convex cores $\mathrm{CC}\left(M_{i}\right)$ converge geometrically to $\mathrm{CC}\left(M_{\infty}\right)$, thus $M_{\infty}$ is doubly degenerate. We can construct a map $f_{\infty}: \bigwedge_{k} \mathbb{S}^{1} \rightarrow M_{\infty}$ by applying the Arzela-Ascoli theorem to the sequence of maps $\phi_{i} \circ f_{i}: \bigwedge_{k} \mathbb{S}^{1} \rightarrow M_{\infty}$, where $\phi_{i}$ is a sequence of almost isometric maps coming from geometric convergence. Clearly $\left(M_{i}, f_{i}\right)$ converges to $\left(M_{\infty}, f_{\infty}\right)$ in $\mathcal{G}$.

Corollary 3.3 Let $M$ be a doubly degenerate $\epsilon$-thick hyperbolic 3-manifold homeomorphic to $\Sigma_{g} \times \mathbb{R}$ and let $p \in M$ be a basepoint. Assume that $\Gamma \subset \pi_{1}(M, p)$ is a proper subgroup that can be generated by $2 g$ loops based at $p$ of length less than $L$. Then $\Gamma$ is convex cocompact and the diameter of the convex core of $\mathbb{H}^{3} / \Gamma$ is bounded above by some constant depending only on $L, \epsilon$ and $g$.

Proof Observe that $\Gamma$ determines an element $(M, f) \in \mathcal{G}=\mathcal{G}(\epsilon, L, 2 g)$, with the extra property that $f$ is not $\pi_{1}$-surjective. Our goal then is to show that if $(M, f) \in \mathcal{G}$ and $f$ is not $\pi_{1}$-surjective, then the diameter of the convex core of the cover $M_{\pi_{1}(f)}$ of $M$ corresponding to the $\pi_{1}$ image of $f$ is bounded above. If not, there is a sequence 
$\left(M_{i}, f_{i}\right)$ of such pairs where these diameters grow without bound. By compactness, we may assume that $\left(M_{i}, f_{i}\right) \rightarrow\left(M_{\infty}, f_{\infty}\right)$ in $\mathcal{G}$. In fact, $f_{\infty}$ cannot be $\pi_{1}$-surjective. To see this, note that by strong convergence there are a compact core $K \subset M_{\infty}$ and almost isometric embeddings $\phi_{i}: K \rightarrow M_{i}$ that are $\pi_{1}$-surjective for large $i$. Therefore, if $f_{\infty}$ is $\pi_{1}$-surjective, then so is $\phi_{i} \circ f_{\infty}$, which is homotopic for large $i$ to $f_{i}$. As $f_{i}$ is never $\pi_{1}$-surjective, it follows that neither is $f_{\infty}$. Lemma 3.1 then shows that $\left(M_{\infty}\right)_{\pi_{1}\left(f_{\infty}\right)}$ is convex-cocompact.

The manifolds $\left(M_{i}\right)_{\pi_{1}\left(f_{i}\right)}$ converge algebraically to $\left(M_{\infty}\right)_{\pi_{1}\left(f_{\infty}\right)}$, and since the limit is convex-cocompact, it follows from [13, Proposition 7.39] that the convergence is strong and the convex cores of the manifolds in the sequence converge geometrically to that of the limit. Consequently, the diameters of these cores must also converge, and therefore must be bounded. This is a contradiction.

\section{Carrier graphs}

In the following, assume $M$ is a closed hyperbolic 3-manifold.

Definition 4.1 A carrier graph for $M$ is a graph $X$ and a map $f: X \rightarrow M$ which induces a surjection on fundamental groups.

Standing assumption In this paper we are interested in generating sets of minimal size, which correspond to carrier graphs with $\operatorname{rank}\left(\pi_{1}(X)\right)=\operatorname{rank}\left(\pi_{1}(M)\right)$. From now on all carrier graphs will be assumed to have this property.

If a carrier graph $f: X \rightarrow M$ is rectifiable, we can pull back path lengths in $M$ to obtain an pseudo-metric on $X$. Collapsing to a point each zero-length segment in $X$ yields a new carrier graph with an actual metric; from now on we will assume all carrier graphs are similarly endowed. Define the length of a carrier graph to be the sum of the lengths of its edges, and a minimal length carrier graph to be a carrier graph which has smallest length (over all carrier graphs of minimal rank). An argument [22] using the Arzela-Ascoli theorem shows that minimal length carrier graphs exist in any closed hyperbolic 3-manifold.

The following Proposition shows that minimal length carrier graphs are geometrically well behaved.

Proposition 4.1 (White [22]) Assume $f: X \rightarrow M$ is a minimal length carrier graph in a closed hyperbolic $3-$ manifold $M$. Then $X$ is trivalent with $2\left(\operatorname{rank}\left(\pi_{1}(M)\right)-1\right)$ vertices and $3\left(\operatorname{rank}\left(\pi_{1}(M)\right)-1\right)$ edges, each edge in $X$ maps to a geodesic segment 
in $M$, the angle between any two adjacent edges is $\frac{2 \pi}{3}$, and the image of any simple closed path in $X$ is an essential loop in $M$.

We conclude this section with a technical result that is instrumental in our proof of Theorem 1.1. A slightly more general theorem was proven by Souto in [17], but the proof given there is somewhat incomplete. We include a full proof of the more general result in Appendix.

Proposition 4.2 (Chains of bounded length) Let $M$ be a closed hyperbolic 3manifold with $f: X \rightarrow M$ a minimal length carrier graph. Then we have a sequence of (possibly disconnected) subgraphs

$$
\varnothing=Y_{0} \subset Y_{1} \subset \ldots \subset Y_{k}=X
$$

such that the length of any edge in $Y_{i+1} \backslash Y_{i}$ is bounded above by some constant depending only on $\operatorname{inj}(M), \operatorname{rank}\left(\pi_{1}(M)\right)$, length $\left(Y_{i}\right)$ and the diameters of the convex cores of the covers of $M$ corresponding to $f_{*}\left(\pi_{1}\left(Y_{i}^{j}\right)\right)$, where $Y_{i}^{1}, \ldots, Y_{i}^{n}$ are the connected components of $Y_{i}$.

\section{Proof of Theorem 1.1}

Fix $\epsilon, g>0$ and assume that $M$ is an $\epsilon$-thick hyperbolic 3-manifold fibering over the circle with fiber $\Sigma_{g}$. The goal of this section is to prove that there are only finitely many such $M$ for which $\operatorname{rank}\left(\pi_{1}(M)\right) \neq 2 g+1$. We begin, however, with a quick computation concerning the girth of $M$.

Definition 5.1 The waist length of $M$, denoted waist $(M)$, is the smallest length of a loop in $M$ that projects nontrivially to $\pi_{1}\left(\mathbb{S}^{1}\right)$.

Proposition 5.1 (Fibered 3-manifolds have high BMI) Let $M$ be an $\epsilon$-thick hyperbolic 3-manifold fibering over the circle with fiber $\Sigma_{g}$. Then

$$
2 \operatorname{diam}(M)-\frac{16}{\epsilon}(2 g-2) \leq \operatorname{waist}(M) \leq 2 \operatorname{diam}(M) .
$$

Proof Assume that $\gamma$ is a loop realizing the waist length of $M$. Canary's Filling Theorem [9] implies that every point in the cyclic cover of $M$ corresponding to the fundamental group of the fiber lies in the image of a simplicial hyperbolic surfaces for which the inclusion map is a homotopy equivalence. Projecting down, this provides an exhaustion of $M$ by simplicial hyperbolic surfaces in the homotopy class of the fiber. By homological considerations, any such surface must intersect $\gamma$. The Bounded 
Diameter Lemma (see Section 2) then implies that $\operatorname{diam}(M) \leq \frac{1}{2}$ waist $(M)+\frac{8}{\epsilon}(2-2 g)$. This establishes the first inequality.

For the second, recall that the fundamental group of $M$ is generated by the set of all loops in $M$ of length less than $2 \operatorname{diam}(M)$. Any generating set for $\pi_{1}(M)$ must contain a loop that projects nontrivially to $\pi_{1}\left(\mathbb{S}^{1}\right)$, so the waist length of $M$ is at most twice its diameter.

There are only finitely many hyperbolic 3-manifolds with diameter less than a given constant [23]. Proposition 5.1 then gives a similar finiteness result for thick hyperbolic 3-manifolds fibering over the circle with a fixed fiber and bounded waist length.

We are now ready to prove the main result of this note.

Theorem 1.1 Given $\epsilon, g>0$ there are at most finitely many $\epsilon$-thick hyperbolic 3-manifolds $M$ fibering over $\mathbb{S}^{1}$ with fiber $\Sigma_{g}$ for which $\operatorname{rank}\left(\pi_{1}(M)\right) \neq 2 g+1$.

Proof Assume that $M$ is an $\epsilon$-thick hyperbolic 3-manifold fibering over the circle with fiber $\Sigma_{g}$ and $\operatorname{rank}\left(\pi_{1}(M)\right) \leq 2 g$. We will show that the waist length of $M$ is bounded by some constant depending only on $\epsilon$ and $g$.

Let $f: X \rightarrow M$ be a minimal length carrier graph. By Proposition 4.2, there is a constant $L$ and a chain of (possibly disconnected) subgraphs

$$
\varnothing=Y_{0} \subset Y_{1} \subset \ldots \subset Y_{k}=X
$$

with length $\left(Y_{i+1}\right)$ bounded above by some constant depending only on $\epsilon, g$, length $\left(Y_{i}\right)$ and the diameters of the convex cores of the covers of $M$ corresponding to the fundamental groups of the connected components of $Y_{i}$.

Assume for the moment that no connected component of $Y_{i}$ runs all the way around the waist of $M$, so that each lifts homeomorphically to the cyclic cover $M_{\pi_{1}\left(\Sigma_{g}\right)}$ of $M$. Since $\operatorname{rank}\left(\pi_{1}(X)\right) \leq 2 g$, the components of $Y_{i}$ have even smaller rank and thus cannot generate the fundamental group of $M_{\pi_{1}\left(\Sigma_{g}\right)}$. Therefore Corollary 3.3 applies to bound the diameters of the associated convex cores in terms of length $\left(Y_{i}\right)$, $\epsilon$ and $g$. It follows that length $\left(Y_{i+1}\right)$ is also bounded above by length $\left(Y_{i}\right), \epsilon$ and $g$.

Applying this argument iteratively, we obtain a length bound for the first subgraph $Y_{i}$ that contains a loop that projects nontrivially to $\pi_{1}\left(\mathbb{S}^{1}\right)$. The length bound depends on $\epsilon, g$ and the index of the subgraph, but since there are at most $3\left(\operatorname{rank}\left(\pi_{1}(M)\right)-1\right)$ edges in $X$ the number of subgraphs in our chain is also limited. Therefore we have that the waist length of $M$ is bounded by a function of $\epsilon$ and $g$. 
Under slight modifications, the proof of Theorem 1.1 shows that for mapping tori with large waist length there is only one Nielsen equivalence class of minimal size generating sets for $\pi_{1}(M)$. The interested reader may compare our proof with [17] for more details.

\subsection{3-Manifolds fibering over an orbifold}

The arguments above also apply to hyperbolic 3-manifolds formed by gluing along their boundaries two twisted interval bundles over a nonorientable surface. One usually says that such a manifold $M$ fibers over the orbifold $\mathbb{S}^{1} /(z \mapsto-z)$. The two embedded copies of the nonorientable surface are called the singular fibers, and the rest of $M$ is foliated by regular fibers, which are orientable and doubly cover the singular fibers. We refer the reader to Hempel [11] for more information on the topology of such manifolds.

If $S_{1}, S_{2} \subset M$ are the singular fibers and $p \in S_{1}$ is a basepoint, then $\pi_{1}(M, p)$ is generated by $\pi_{1}\left(S_{1}, p\right)$ and any loop freely homotopic into $S_{2}$, but not into $S_{1}$. Using this, one can check that if the regular fibers have genus $g$, then

$$
\operatorname{rank}\left(\pi_{1}(M)\right) \leq g+2 .
$$

We then have the following analogue of Theorem 1.1:

Theorem 5.2 Given $\epsilon, g>0$, there are at most finitely many hyperbolic 3-manifolds $M$ fibering over $\mathbb{S}^{1} /(z \mapsto-z)$ with regular fiber $\Sigma_{g}$ for which $\operatorname{rank}\left(\pi_{1}(M)\right) \neq g+2$.

The proof is nearly the same. One takes a minimal length carrier graph and shows that if the distance between the two singular fibers is large enough then one of the subgraphs given by Proposition 4.2 fills out the fundamental group of one of the singular fibers of $M$. This forces the fundamental group of the subgraph to have rank at least $g+1$, implying that the carrier graph has rank at least $g+2$. The finiteness statement follows because an upper bound for the distance between the two singular fibers provides a upper bound on the diameter, at worst by imitating the argument in Proposition 5.1.

\section{Appendix Chains of bounded length}

We prove here the generalization of Proposition 4.2 promised in Section 4. The idea of the proof given below was originally sketched by Souto in [17]; the purpose of this Appendix is to fill in some missing details. 
Assume that $M=\mathbb{H}^{3} / \Gamma$ is a closed hyperbolic 3-manifold and $f: X \rightarrow M$ is a minimal length carrier graph. Choose an edge $e \subset X$ and a subgraph $Y \subset X$. Our first goal will be to provide a useful definition of the length of $e$ relative to the subgraph $Y$. This should vanish when $e \subset Y$ and should agree with the hyperbolic length of $f(e)$ when neither of the vertices of $e$ lies inside $Y$. If $X$ is embedded as a subset of $M$ with $f$ the inclusion map, then relative length is similar to the length $e$ has outside of the hyperbolic convex hulls of the components of $Y$ that $e$ touches, but we need to do our measurements in the universal cover and throw out sections of $e$ that lie inside some of the thin parts of $M$.

To clarify this, fix a universal covering $\pi_{X}: \tilde{X} \rightarrow X$ and a lift $\tilde{f}: \tilde{X} \rightarrow \mathbb{H}^{3}$ of $f$. Assume that a vertex $v$ of $e$ lies in a connected component $Z_{v} \subset Y$ and choose lifts $\widehat{e}, \widehat{Z_{v}} \subset \tilde{X}$ of $e$ and $Z$ that touch above $v$. Let $\Gamma \tilde{f}\left(\widehat{Z_{v}}\right)$ be the subgroup of $\Gamma$ that leaves $\tilde{f}\left(\widehat{Z_{v}}\right)$ invariant.

Definition A.1 (Thick convex hulls) The thick convex hull of $\tilde{f}\left(\widehat{Z_{v}}\right)$, denoted by $\operatorname{TCH}\left(\tilde{f}\left(\widehat{Z_{v}}\right)\right)$, is the radius-1 neighborhood of the smallest convex set $K$ containing $\widetilde{f}\left(\widehat{Z_{v}}\right)$ such that for every $\gamma \in \Gamma \widetilde{f}\left(\widehat{Z_{v}}\right)$ and $x \in \mathbb{H}^{3} \backslash K$, we have $\mathrm{d}(\gamma(x), x) \geq 1$.

Definition A.2 (Edge length relative to a subgraph) Define the length of e relative to $Y$, denoted length ${ }_{Y}(e)$, to be the length of the part of $\tilde{f}(\hat{e})$ that lies outside of $\operatorname{TCH}\left(\tilde{f}\left(\widehat{Z_{v}}\right)\right)$ for each vertex $v$ of $e$ contained in $Y$.

It is easy to see that the relative length of $e$ is well-defined, independent of the lifts chosen above. The definition is a bit less complicated if we assume that $X$ is embedded as a subset of $M$. For then we can lift $e$ directly to $\mathbb{H}^{3}$ along with any connected components of $Y$ that $e$ touches, and then measure the length of $e$ 's lift outside of the thick convex hulls of the lifted subgraphs. In the proofs below, we will assume $X$ to be embedded in order to remove a level of notational hinderance. The arguments will be exactly the same in the general case.

Although an edge can have very long absolute length while having short length relative to a subgraph $Y$, we can bound this difference if we have some control over the geometry of the covers of $M$ corresponding to the fundamental groups of the components of $Y$.

Lemma A.1 Assume that $M$ is a closed hyperbolic 3-manifold, $f: X \rightarrow M$ is a minimal length carrier graph, $Y$ is a subgraph of $X$ and $e$ is an edge of $X \backslash Y$. Then length $(e)$ is bounded above by a constant depending only on length $Y_{Y}(e)$, length $(Y)$, $\operatorname{inj}(M), \operatorname{rank}\left(\pi_{1}(M)\right)$ and the diameters of the convex cores of the covers of $M$ corresponding to the components of $Y$ that e touches. 
Proof As mentioned above, we forget about $f$ and assume that $X$ is embedded as a subset of $M$. Suppose that $e$ shares a vertex with a connected component $Z \subset Y$, and let $\tilde{e}, \tilde{Z} \subset \mathbb{H}^{3}$ be lifts that touch above that vertex. Since $X$ is minimal length, $\widetilde{e} \cap \operatorname{TCH}(\widetilde{Z})$ must minimize the distance from $\widetilde{e} \cap \partial \operatorname{TCH}(\widetilde{Z})$ to $\tilde{Z}$. For otherwise, one could replace it by a minimizing segment; extending equivariantly gives a new carrier graph homotopic to $X$ and of smaller length, violating the minimality assumption. Thus a bound on the Hausdorff distance between $\widetilde{Z}$ and $\operatorname{TCH}(\tilde{Z})$ limits the length that $\tilde{e}$ can have inside of $\operatorname{TCH}(\tilde{Z})$, and we will show that this is bounded in terms of the quantities mentioned in the statement of the Lemma.

We first claim that the hyperbolic distance from $\tilde{Z}$ to $\mathrm{CH}\left(\Lambda\left(\Gamma_{\tilde{Z}}\right)\right)$ is bounded above by a constant depending only on $\operatorname{inj}(M)$ and $\operatorname{rank}\left(\pi_{1}(M)\right)$. Choose an infinite piecewise geodesic path $\gamma \subset \widetilde{Z}$ that projects to a simple closed curve in $Y$ and let $g \in \Gamma_{\widetilde{Z}}$ be the corresponding deck transformation. Taking a maximal sequence of consecutive edges of $\gamma$ that project to distinct edges in $M$ yields a subpath $\gamma^{\prime}$ whose $g$-translates cover $\gamma$. Note that the orthogonal projection of $\gamma^{\prime}$ to axis $(g)$ has length equal to the translation distance of $g$, which is at least $\operatorname{inj}(M)$. By Proposition 4.1, $X$ has $3\left(\operatorname{rank}\left(\pi_{1}(M)\right)-1\right)$ edges; the number of edges in $\gamma^{\prime}$ can certainly be no greater than this. Thus there is an edge of $\gamma$ whose orthogonal projection to axis $(g)$ has length at least $\operatorname{inj}(M) /\left(3\left(\operatorname{rank}\left(\pi_{1}(M)\right)-1\right)\right)$. It follows from elementary hyperbolic geometry that there is a point on this edge whose distance from axis $(g)$ is bounded above by a constant depending on that length. For instance, if one draws two lines $l_{1}, l_{2}$ orthogonal to $\operatorname{axis}(g)$ that $\operatorname{are} \operatorname{inj}(M) /\left(3\left(\operatorname{rank}\left(\pi_{1}(M)\right)-1\right)\right)$ apart, then the distance from the edge to $\operatorname{axis}(g)$ is at most the distance to $\operatorname{axis}(g)$ from either of the two geodesic lines that share one endpoint on $\mathbb{S}_{\infty}^{1}$ with $l_{1}$, the other with $l_{2}$, and lie on one side of $\operatorname{axis}(g)$. This proves the claim.

Now $\widetilde{Z}$ and $\operatorname{CH}\left(\Lambda\left(\Gamma_{\tilde{Z}}\right)\right)$ are both invariant under the action of $\Gamma_{\widetilde{Z}}$ with quotients of bounded diameter, so our limit on the hyperbolic distance between them translates into a bound on their Hausdorff distance. But if $\tilde{Z}$ is Hausdorff-close to a convex set then it must also be Hausdorff-close to its convex hull, $\mathrm{CH}(\widetilde{Z})$. Since the Hausdorff distance from $\mathrm{CH}(\tilde{Z})$ to $\mathrm{TCH}(\widetilde{Z})$ is controlled by $\operatorname{inj}(M)$, we have a bound on the Hausdorff distance between $\widetilde{Z}$ and $\operatorname{TCH}(\widetilde{Z})$.

For a subgraph $Z \subset X$, we define the length of $Z$ relative to $Y$ to be

$$
\text { length }_{Y}(Z)=\sum_{\text {edges } e \subset Z} \text { length }_{Y}(e) .
$$

Using our definition of relative length, we can streamline the formulation of Proposition 4.2. The statement given earlier follows from this one after applying Lemma A.1. 
Proposition A.2 (Chains of bounded length) There is a universal constant $L$ with the property that if $M$ is a closed hyperbolic 3-manifold and $f: X \rightarrow M$ is a minimal length carrier graph then we have a sequence of (possibly disconnected) subgraphs

$$
\varnothing=Y_{0} \subset Y_{1} \subset \ldots \subset Y_{k}=X
$$

such that length $Y_{i}\left(Y_{i+1}\right)<L$ for all $i$.

Proof It is a standard fact in hyperbolic geometry that there exist a universal constant $C>0$ with the following property:

(1) any path in $\mathbb{H}^{3}$ made of geodesic segments of length at least $C$ connected with angles at least $\frac{\pi}{3}$ is a quasi-geodesic.

There is also a constant $D>C$ such that

(2) if $N \subset \mathbb{H}^{3}$ contains the axis of a hyperbolic isometry $\gamma$ and $\mathrm{d}(x, \gamma(x)) \geq 1$ for all $x \in \mathbb{H}^{3} \backslash N$, then $\mathrm{d}(x, \gamma(x)) \geq C$ for all $x \in \mathbb{H}^{3} \backslash \mathcal{N}_{D}(N)$

(3) any geodesic ray emanating from a convex subset $K \subset \mathbb{H}^{3}$ that leaves $\mathcal{N}_{D}(K)$ meets $\partial \mathcal{N}_{D}(K)$ in an angle of at least $\frac{\pi}{3}$,

and finally a constant $B>0$ for which

(4) any geodesic exiting the radius-1 neighborhood of a convex subset $K \subset \mathbb{H}^{3}$ will exit $\mathcal{N}_{D}(K)$ after an additional length less than $B$.

We will show that if $Y$ is any subgraph of $X$ then there is an edge in $X \backslash Y$ of length at most $L=C+2 B$ relative to $Y$; applying this iteratively will give the chain of subgraphs in the statement of the Proposition.

So, suppose that $Y$ is a subgraph of $X$. Observe that since the fundamental group of a closed hyperbolic manifold cannot be free, there is an essential closed loop $\gamma \subset X$ that is nullhomotopic in $M$. Furthermore, since $\pi_{1}(M)$ does not split as a free product [11, Theorem 7.1] we can pick $\gamma$ so that it has no subpath contained entirely in $Y$ that is also a closed loop nullhomotopic in $M$. Lifting $\gamma$ to $\mathbb{H}^{3}$ then gives a closed loop $\tilde{\gamma} \subset \mathbb{H}^{3}$ such that each time $\tilde{\gamma}$ touches a component of $\pi_{M}^{-1}(Y)$ it enters and leaves that component using different edges of $\pi_{M}^{-1}(X \backslash Y)$.

The first crucial observation is that one of the edges of $\gamma$ must have length less that $L$. For otherwise, $\tilde{\gamma}$ is a closed path in $\mathbb{H}^{3}$ made up of geodesic segments of length at least $L$ connected at $\frac{\pi}{3}$-angles, which is impossible by definition of $L$. If this short edge lies outside $Y$, then we are done. However, it very well might not, so in the remainder of the proof we will develop a version of this argument that runs relative 
to $Y$. This will produce a short edge outside $Y$, but we will be forced to measure its length relatively rather than absolutely.

So, consider a maximal segment of $\tilde{\gamma}$ that is contained in a component $\tilde{Z}$ of $\pi_{M}^{-1}(Y)$ and let $e$ and $f$ be the edges that $\tilde{\gamma}$ traverses before and after the segment in $\tilde{Z}$. If $e$ or $f$ has length less than $L$ relative to $Y$, then we are done. Otherwise, the two edges have a length of at least $L$ left after exiting $\operatorname{TCH}(\tilde{Z})$, so by (4) both of these edges must exit $\mathcal{N}_{D}(\mathrm{TCH}(\tilde{Z}))$; let $e_{0}$ and $f_{0}$ be the points where they meet $\partial \mathcal{N}_{D}(\mathrm{TCH}(\widetilde{Z}))$. Assume for the moment that the distance between $e_{0}$ and $f_{0}$ is less than $C$. Then by (2), $e$ and $f$ project to different edges in $X$. Substituting $\pi_{M}\left(e \cap \mathcal{N}_{D}(\mathrm{TCH}(\widetilde{Z}))\right) \subset X$ with the projection of the geodesic between $e_{0}$ and $f_{0}$ therefore yields a new carrier graph for $M$, and since the new edge has length less than $C$ while the old has length at least $D$ our new carrier graph has shorter length than $X$. This contradicts the minimality of $X$, so $\mathrm{d}\left(e_{0}, f_{0}\right) \geq C$.

We can now create a new closed path in $\mathbb{H}^{3}$ from $\tilde{\gamma}$ as follows: each time $\tilde{\gamma}$ traverses a component $\tilde{Z}$ of $\pi_{M}^{-1}(Y)$, replace the part of $\tilde{\gamma}$ that lies inside $\mathcal{N}_{D}(\mathrm{TCH}(\tilde{Z}))$ by the geodesic with the same endpoints. Then the new path is composed of geodesic segments of length at least $C$, and by (3), the segments intersect with angles at least $\frac{\pi}{3}$. Therefore it is a quasi-geodesic. Since it is also closed, this is impossible.

\section{References}

[1] I Agol, Tameness of hyperbolic 3-manifolds arXiv:math.GT/0405568

[2] L V Ahlfors, Conformal invariants: topics in geometric function theory, McGraw-Hill Ser. in Higher Math., McGraw-Hill, New York (1973) MR0357743

[3] D Bachman, S Schleimer, Surface bundles versus Heegaard splittings, Comm. Anal. Geom. 13 (2005) 903-928 MR2216145

[4] R Benedetti, C Petronio, Lectures on hyperbolic geometry, Universitext, Springer, Berlin (1992) MR1219310

[5] S A Bleiler, C D Hodgson, Spherical space forms and Dehn filling, Topology 35 (1996) 809-833 MR1396779

[6] F Bonahon, Bouts des variétés hyperboliques de dimension 3, Ann. of Math. (2) 124 (1986) 71-158 MR847953

[7] D Calegari, D Gabai, Shrinkwrapping and the taming of hyperbolic 3-manifolds, J. Amer. Math. Soc. 19 (2006) 385-446 MR2188131

[8] R D Canary, Ends of hyperbolic 3-manifolds, J. Amer. Math. Soc. 6 (1993) 1-35 MR1166330 
[9] RD Canary, A covering theorem for hyperbolic 3-manifolds and its applications, Topology 35 (1996) 751-778 MR1396777

[10] B Farb, L Mosher, Convex cocompact subgroups of mapping class groups, Geom. Topol. 6 (2002) 91-152 MR1914566

[11] J Hempel, 3-Manifolds, Ann. of Math. Studies 86, Princeton Univ. Press (1976) MR0415619

[12] R Kent, C Leininger, Shadows of mapping class groups: capturing convex cocompactness arXiv:math.GT/0505114

[13] K Matsuzaki, M Taniguchi, Hyperbolic manifolds and Kleinian groups, Oxford Math. Monog., Oxford Science Publ., The Clarendon Press, Oxford Univ. Press, New York (1998) MR1638795

[14] K Ohshika, The continuity of convex cores with respect to the geometric topology, Comm. Anal. Geom. 13 (2005) 479-510 MR2171689

[15] K Rafi, A characterization of short curves of a Teichmüller geodesic, Geom. Topol. 9 (2005) 179-202 MR2115672

[16] G P Scott, G A Swarup, Geometric finiteness of certain Kleinian groups, Proc. Amer. Math. Soc. 109 (1990) 765-768 MR1013981

[17] J Souto, Rank and topology of hyperbolic 3-manifolds, I, Preprint

[18] J Souto, The rank of the fundamental group of certain hyperbolic 3-manifolds fibering over the circle, from: "The Zieschang Gedenkschrift”, (M Boileau, M Scharlemann, R Weidmann, editors), Geom. Topol. Monogr. 14, Geom. Topol. Publ., Coventry (2008) 505-518

[19] W P Thurston, Hyperbolic structures on 3-manifolds, II: Surface groups and 3manifolds fibering over the circle arXiv:math.GT/9801045

[20] W P Thurston, The geometry and topology of three-manifolds, Princeton Univ. Math. Dept. Lecture Notes (1979) Available at http://msri.org/publications/books/ gt $3 \mathrm{~m} /$

[21] F Waldhausen, On irreducible 3-manifolds which are sufficiently large, Ann. of Math. (2) 87 (1968) 56-88 MR0224099

[22] ME White, Injectivity radius and fundamental groups of hyperbolic 3-manifolds, Comm. Anal. Geom. 10 (2002) 377-395 MR1900756

[23] R Young, Counting hyperbolic manifolds with bounded diameter, Geom. Dedicata 116 (2005) 61-65 MR2195441

Department of Mathematics, University of Chicago, Chicago, IL 60637, USA

biringer@math. uchicago . edu

http://www. math.uchicago.edu/ biringer

Received: 11 June 2008 Revised: 7 September 2008 